\title{
A FINITE DIMENSIONAL ALGEBRA OF THE DIAGRAM OF A KNOT
}

\author{
CLAUDE CIBILS
}

(Communicated by Birge Huisgen-Zimmermann)

\begin{abstract}
To a regular projection of a knot we associate a finite dimensional non-commutative associative algebra which is self-injective and special biserial.
\end{abstract}

\section{INTRODUCTION}

Let $D$ be an oriented knot diagram that is a regular projection of a knot to the plane where each crossing is given with the information of which part of the knot under-crosses the other one; this appears in $D$ by interrupting the part of the diagram which is under-crossing.

In this note we associate to $D$ a finite dimensional algebra over a field $k$, presented by a quiver with relations deduced from the knot. In other words we associate to $D$ a $k$-category given by a presentation; it has a finite number of objects corresponding to the crosses, and finite dimensional vector spaces of morphisms. The algebra of the diagram is the direct sum of all the morphisms of the $k$-category with product induced by the composition of the category or, equivalently, is the path algebra of the quiver modulo the two-sided ideal generated by the relations.

This algebra of the knot projection is Morita reduced, special biserial and selfinjective. As such it is not invariant under Reidemeister moves since its dimension changes.

The main purpose of this note is the description of this family of algebras. They can be of interest in order to test homological conjectures or to analyze representation theory aspects. On the other hand one may expect information on the knot via the algebra of a diagram, as well as links between knot theory and the representation theory of finite dimensional algebras.

In some examples this algebra happens to admit a connected grading by the fundamental group of the knot.

\section{QUIVER AND RELATIONS OF AN ORIENTED KNOT DIAGRAM}

A quiver is an oriented graph $Q$ given by two finite sets $Q_{0}$ (vertices) and $Q_{1}$ (arrows) and two maps $s, t: Q_{1} \rightarrow Q_{0}$ assigning to each arrow $a$ the source and target vertices $s(a)$ and $t(a)$. Consider the vector space $k Q$ with basis the set of oriented paths of $Q$ including the trivial ones given by the vertices. The quiver path algebra is $k Q$ equipped with the product on paths induced by their concatenation

Received by the editors December 6, 2012.

2010 Mathematics Subject Classification. Primary 16S99, 57M25.

(c) 2014 American Mathematical Society 
if it can be performed and 0 otherwise. This way the vertices provide a complete set of primitive orthogonal idempotents. Note that $Q_{0}$ needs to be non-empty in order to get an algebra.

Consider $F$ the two-sided ideal generated by the arrows. Clearly $k Q / F \simeq k Q_{0}$, where $k Q_{0}$ is the commutative semi-simple algebra of the set $Q_{0}$. In case $Q$ has no oriented cycles $F$ is the Jacobson radical of $k Q$. A well known theorem of P. Gabriel states that for each finite dimensional Morita reduced algebra $\Lambda$ over an algebraically closed field there is a unique quiver $Q_{\Lambda}$ such that $\Lambda$ is isomorphic to a quotient $k Q_{\Lambda} / I$ where $I$ is an admissible two-sided ideal; that is, $I$ contains $F^{2}$ and is contained in $F^{n}$ for some positive integer $n$. Note that $I$ is not unique in general, while $Q_{\Lambda}$ is the Ext quiver with vertices the iso-classes of simple modules and as many arrows between two simples than the dimension of $\mathrm{Ext}_{\Lambda}^{1}$ between them.

Let $D$ be an oriented knot diagram. An arc of $D$ is obtained by following the diagram according to its orientation from an under-crossing to the next one; in other words, an arc is a connected component of $D$. A segment of the diagram is obtained by following the diagram according to its orientation, from one crossing to a crossing with no crossings in-between.

Definition 2.1. The quiver $\mathbf{Q}_{\mathbf{D}}$ has set of vertices the crossings of $D$. Note that this requires having at least one crossing in $D$ so that $\left(Q_{D}\right)_{0} \neq \emptyset$. Each segment provides an arrow having source and target the corresponding crossings. We say that the source of an arrow is negative or positive according to whether the segment starts respectively by under-crossing or by over-crossing. Similarly the target vertex of an arrow is also negative or positive.

Remark 2.2. There are two specific oriented cycles at each vertex $e$ as follows:

(1) $\alpha_{e}$ starts at $e$ by the arrow with positive source $e$ and successive arrows by browsing the oriented diagram until reaching the arrow having target $e$ with negative sign.

(2) Similarly, $\beta_{e}$ starts at $e$, but by the negative source arrow, and ends with the arrow with positive target $e$.

Definition 2.3. The two fundamental cycles at $e$ are as follows: the overcrossing (or positive) one, $\gamma_{e}^{+}=\beta_{e} \alpha_{e}$, and the under-crossing (or negative) one, $\gamma_{e}^{-}=\alpha_{e} \beta_{e}$.

Lemma 2.4. The length of the fundamental cycles does not depend on the vertex and equals the number of arrows $n_{D}$ of $Q_{D}$.

Let $\tau: Q_{0} \rightarrow k^{\bullet}$ be a map. For instance, $\tau(e)=q^{l(e)}$, where $q \in k^{\bullet}$ and $l: Q_{0} \rightarrow \mathbb{Z}$ is some map given for example by the length of $\alpha_{e}$. We consider a two-sided ideal $I_{\tau}$ of $k Q_{D}$ generated by two kinds of relations as follows:

- Type I : Let $b a$ be a path of length 2 in $Q_{D}$ and let $e=t(a)=s(b)$ be its middle vertex. In case the signs at $e$ of $a$ and $b$ are different (that is, if $b a$ is not a follow-up of two segments of the diagram), then $b a$ is a generator. This way each vertex of the quiver provides two generators.

- Type II : The elements $\alpha_{e} \beta_{e}-\tau(e) \beta_{e} \alpha_{e}$ for all the vertices $e$.

Definition 2.5. The algebra of the diagram $D$ with respect to $\tau$ is $\Lambda_{D, \tau}=$ $k Q_{D} / I_{\tau}$. 
Proposition 2.6. The algebra $\Lambda_{D, \tau}$ is finite dimensional. A basis is given by the positive fundamental cycles and the non-zero paths of length strictly less than $n_{D}$, that is, the paths made by following-up the segments and having strictly less than $n_{D}$ segments.

Proof. The proof relies on the observation that a path $\delta$ of length $n_{D}+1$ is zero in the algebra. Indeed, let $\delta=a \gamma$ where $\gamma$ is a fundamental cycle. If the source sign of $a$ is not the same as the target sign of $\gamma$, then $\delta \in I_{\tau}$. Otherwise let $\gamma^{\prime}$ be the other fundamental cycle; then $a \gamma$ and $a \gamma^{\prime}$ are equal up to a non-zero element of $k$ and the latter is in the ideal.

Remark 2.7. The number of vertices of $Q$ change through the first and second Reidemeister moves.

Let $J_{D}$ be the two-sided ideal generated by the relations of type I and type II', where type II' is the set of all paths of length $n_{D}+1$. Instead of $\Lambda_{D, \tau}$ a monomial algebra $\Xi_{D}=k Q_{D} / J_{D}$ can be considered which we call the monomial algebra of the diagram. A larger basis than before is provided by all the non-zero paths of length strictly less than $n_{D}+1$.

Example 2.8. Let $D$ be the diagram of the trivial knot with one crossing. Then the algebra $\Lambda_{D}, \tau$ is $k\{a, b\} /\langle a b-q b a\rangle$ for $q \in k^{\bullet}$. This algebra provides the first example for a negative answer to Happel's question; see [3. More precisely its global dimension is infinite, but for $q$ not a root of unity it has zero Hochschild cohomology in degrees large enough (in fact starting at degree 3). Nevertheless this algebra verifies Han's conjecture (see [16]); namely, its Hochschild homology is non-zero in arbitrarily large degrees (in fact in all degrees).

\section{Properties}

We recall first the definition of a family of algebras which arose in representation theory of finite dimensional algebras.

Definition 3.1. Let $Q$ be a quiver and $I$ a two-sided admissible ideal generated by a set of relation $\rho$. Then $(Q, \rho)$ is called special biserial (see for instance [13]) if it verifies the following conditions:

(1) Any vertex of $Q$ is the source of at most two arrows and is the target of at most two arrows.

(2) If two different arrows $c$ and $d$ start at the target of an arrow $a$, then at least one of the paths $c a$ or $d a$ is in $\rho$.

(3) If two different arrows $a$ and $b$ end at the source of an arrow $c$, then at least one of the two paths $c a$ or $c b$ is in $\rho$.

As mentioned by C.M. Ringel in [13] special biserial algebras were first considered by I.M. Gelfand and V.A. Ponomarev in [15. Blocks of a group algebra with cyclic or dihedral defect group are special biserial. As a consequence of [15] special biserial algebras are of tame representation type; in other words their indecomposable modules can be classified (see also [12,23]). Precise conditions are given in [1] for the vanishing of the first Hochschild cohomology of a special biserial algebra (which in turn implies that the cohomology in degrees larger than 1 also vanishes). 
The following result is immediate:

Proposition 3.2. The algebra (or the monomial algebra) of the diagram of a knot is special biserial.

We recall that an algebra is self-injective if it admits a non-degenerate bilinear form $\beta: \Lambda \times \Lambda \rightarrow k$ which is associative, that is, $\beta(x y, z)=\beta(x, y z)$ for any triple of elements $(x, y, z)$ in $\Lambda$. Associated to $\beta$ there is a linear map $t: \Lambda \rightarrow k$ given by $t(x)=\beta(x, 1)$ which is a free generator of the left $\Lambda$-module $\operatorname{Hom}_{k}(\Lambda, k)$.

Theorem 3.3. Algebras of diagrams of knots are self-injective.

Proof. Let $\delta$ be a positive length basis path of $\Lambda_{D, \tau}$ (according to Proposition 2.6) with source vertex $e$. We define $\delta^{\prime}$ to be the path such that $\delta^{\prime} \delta=\gamma_{e}^{\epsilon}$, where $\gamma_{e}^{\epsilon}$ is the fundamental cycle and $\epsilon$ is the sign of $e$ at the first arrow of $\delta$. Note that $\left(\gamma_{e}^{+}\right)^{\prime}=e$. Moreover, for each vertex $e$ we put $e^{\prime}=\gamma_{e}^{+}$.

In case $\delta_{1}$ and $\delta_{2}$ are basis paths such that $\delta_{2} \neq \delta_{1}^{\prime}$, we put $\beta\left(\delta_{2}, \delta_{1}\right)=0$.

If $\delta_{2}=\delta_{1}^{\prime}$ we consider two cases:

(1) In case $\delta_{1}$ is a vertex or if the source of the first arrow of $\delta_{1}$ is positive, then $\beta\left(\delta_{1}^{\prime}, \delta_{1}\right)=1$.

(2) If the sign of the source $e$ of the first arrow of $\delta_{1}$ is negative, then $\beta\left(\delta_{1}^{\prime}, \delta_{1}\right)=$ $\tau(e)$.

The only difficulty for verifying that $\beta$ is associative arises when $\delta_{1}$ has a first arrow with negative source $e$. We need to prove that

$$
\beta\left(\delta_{1}^{\prime}, \delta_{1}\right)=\beta\left(\delta_{1}^{\prime} \delta_{1}, e\right) .
$$

We have defined $\beta\left(\delta_{1}^{\prime}, \delta_{1}\right)=\tau(e)$ while

$$
\beta\left(\delta_{1}^{\prime} \delta_{1}, e\right)=\beta\left(\gamma_{e}^{-}, e\right)=\beta\left(\tau(e) \gamma_{e}^{+}, e\right)=\tau(e) \beta\left(\gamma_{e}^{+}, e\right)=\tau(e) .
$$

There is no difficulty for showing that $\beta$ is non-degenerated.

Remark 3.4. The class of special biserial self-injective algebras has been studied by K. Erdmann and A. Skowroński with respect to Euclidian components of the stable Auslander-Reiten quiver; see [14. See also the work by Z. Pogorzaly [21] concerning stable equivalence of this class of algebras. Precise computations of the Hochschild cohomology of certain self-injective special biserial algebras are performed in [22].

\section{GRADings}

The fundamental group à la Grothendieck of a $k$-category has been considered in [7-[10, 11]. Previously a fundamental group depending on a presentation by a quiver with relations has been studied in relation with representation theory; see for instance 2, 4, 5, 17 20]. The main tool for the theory of the intrinsic fundamental group are the connected gradings as follows.

Definition 4.1. Let $\mathcal{B}$ be a small $k$-category. A grading $X$ of $\mathcal{B}$ with structural group $\Gamma(X)$ is firstly a direct sum decomposition of each vector space of morphisms indexed by elements of $\Gamma(X)$ - a direct summand with index $g$ of this decomposition is called a homogeneous component of degree $g$, and a non-zero morphism in this component is said to be homogeneous of degree $g$. Secondly, the composition of two homogeneous morphisms is homogeneous with degree the product of the degrees. 
The precise definition of homogeneous walks is given for instance in 11]. Roughly each homogeneous morphism $\varphi$ of degree $g$ provides a virtual one $(\varphi,-1)$ with reversed source and target vertices and of settled degree $g^{-1}$. A homogeneous walk is a sequence of concatenated (virtual or not) homogeneous morphisms; its degree is the product of the degrees.

The grading $X$ is connected if between two fixed objects and for any element of $g \in \Gamma(X)$ there exists a homogenous walk relying on the objects having degree $g$. In this case the smash product provides a connected category which is a Galois covering of $\mathcal{B}$; see [6].

The fundamental group is obtained by considering all the connected gradings of $\mathcal{B}$ and coherent families of elements of the structure groups with respect to morphisms of gradings; see 11 .

We recall the following convention: a crossing of $D$ is positive if following the diagram according to the orientation the under line of the diagram goes from right to left. It is negative otherwise.

Let $D$ be the diagram of an oriented knot. Recall that the fundamental group of the complement of the knot with base point at the infinity is generated by the loop which passes just under each portion of the knot corresponding to an arc of the projection.

Definition 4.2. Let $Q_{D}$ be the diagram of a knot. The grading of $Q_{D}$ by the fundamental group of the knot is as follows: given an arrow, consider the corresponding segment and the arc to which it belongs. In case the crossings at the vertices of the arrow have the same sign, the degree of the arrow is the generator of the fundamental group corresponding to the arc. Otherwise the degree is trivial.

The verification of the following result is not difficult using the Wirtinger presentation of the fundamental group of the knot.

Example 4.3. For the usual diagram of the trefoil knot or of the figure-height knot the two-sided ideal $I_{\tau}$ is homogeneous, and the resulting grading for the algebra of the diagram is connected.

Nevertheless it seems that for the diagram of the knot $6_{-} 3$ (see for instance the Knot Atlas) the ideal is not homogeneous.

\section{ACKNOWLEDGEMENTS}

The author thanks the organizers and lecturers of the CIMPA research school Symplectic Geometry and Geometric Topology, Meknès (Morocco) in 2012, which was the inspiration for the content of this note.

\section{REFERENCES}

[1] Ibrahim Assem, Juan Carlos Bustamante, and Patrick Le Meur, Special biserial algebras with no outer derivations, Colloq. Math. 125 (2011), no. 1, 83-98, DOI 10.4064/cm125-1-6. MR2860583 (2012k:16025)

[2] K. Bongartz and P. Gabriel, Covering spaces in representation-theory, Invent. Math. 65 (1981/82), no. 3, 331-378, DOI 10.1007/BF01396624. MR643558(84i:16030)

[3] Ragnar-Olaf Buchweitz, Edward L. Green, Dag Madsen, and Øyvind Solberg, Finite Hochschild cohomology without finite global dimension, Math. Res. Lett. 12 (2005), no. 5-6, 805-816. MR2189240 (2006k:16019)

[4] Juan Carlos Bustamante, On the fundamental group of a Schurian algebra, Comm. Algebra 30 (2002), no. 11, 5307-5329, DOI 10.1081/AGB-120015654. MR.1945091(2004d:16025) 
[5] Juan Carlos Bustamante and Diane Castonguay, Fundamental groups and presentations of algebras, J. Algebra Appl. 5 (2006), no. 5, 549-562, DOI 10.1142/S021949880600179X. MR2269406 (2007i:16027)

[6] Claude Cibils and Eduardo N. Marcos, Skew category, Galois covering and smash product of a $k$-category, Proc. Amer. Math. Soc. 134 (2006), no. 1, 39-50 (electronic), DOI 10.1090/S00029939-05-07955-4. MR2170541 (2006e:18003)

[7] Claude Cibils, María Julia Redondo, and Andrea Solotar, Connected gradings and the fundamental group, Algebra Number Theory 4 (2010), no. 5, 625-648, DOI 10.2140/ant.2010.4.625. MR2679101 (2011m:16088)

[8] Claude Cibils, María Julia Redondo, and Andrea Solotar, Fundamental group of Schurian categories and the Hurewicz isomorphism, Doc. Math. 16 (2011), 581-595. MR2837545

[9] Claude Cibils, María Julia Redondo, and Andrea Solotar, The intrinsic fundamental group of a linear category, Algebr. Represent. Theory 15 (2012), no. 4, 735-753, DOI 10.1007/s10468010-9263-1. MR 2944440

[10] Claude Cibils, Maria Julia Redondo, and Andrea Solotar, Full and convex linear subcategories are incompressible, Proc. Amer. Math. Soc. 141 (2013), no. 6, 1939-1946, DOI 10.1090/S0002-9939-2013-11470-X. MR3034421

[11] C. Cibils, M. J. Redondo, and A. Solotar, On universal gradings, versal gradings and Schurian generated categories. arXiv:1210.4098v1. To appear in J. Noncommut. Geom.

[12] Piotr Dowbor and Andrzej Skowroński, Galois coverings of representation-infinite algebras, Comment. Math. Helv. 62 (1987), no. 2, 311-337, DOI 10.1007/BF02564450. MR896100 (88m:16020)

[13] Claus Michael Ringel, The minimal representation-infinite algebras which are special biserial, Representations of algebras and related topics, EMS Ser. Congr. Rep., Eur. Math. Soc., Zürich, 2011, pp. 501-560, DOI 10.4171/101-1/11. MR2931904

[14] Karin Erdmann and Andrzej Skowroński, On Auslander-Reiten components of blocks and self-injective biserial algebras, Trans. Amer. Math. Soc. 330 (1992), no. 1, 165-189, DOI 10.2307/2154159. MR1144759 (93b:16022)

[15] I. M. Gel'fand and V. A. Ponomarev, Indecomposable representations of the Lorentz group (Russian), Uspehi Mat. Nauk 23 (1968), no. 2 (140), 3-60. MR0229751 (37 \#5325)

[16] Yang Han, Hochschild (co)homology dimension, J. London Math. Soc. (2) 73 (2006), no. 3, 657-668, DOI 10.1112/S002461070602299X. MR2241972 (2007c:16018)

[17] Patrick Le Meur, The universal cover of an algebra without double bypass, J. Algebra 312 (2007), no. 1, 330-353, DOI 10.1016/j.jalgebra.2006.10.035. MR2320460 (2008c:16026)

[18] Patrick Le Meur, The fundamental group of a triangular algebra without double bypasses (English, with English and French summaries), C. R. Math. Acad. Sci. Paris 341 (2005), no. 4, 211-216, DOI 10.1016/j.crma.2005.07.004. MR2164674 (2006k:16033)

[19] P. Le Meur, Revêtements galoisiens et groupe fondamental d'algèbres de dimension finie, Ph.D. thesis, Université Montpellier 2, 2006. http://tel.archives-ouvertes.fr/ tel-00011753

[20] R. Martínez-Villa and J. A. de la Peña, The universal cover of a quiver with relations, J. Pure Appl. Algebra 30 (1983), no. 3, 277-292, DOI 10.1016/0022-4049(83)90062-2. MR724038 (85f:16035)

[21] Zygmunt Pogorzały, Algebras stably equivalent to self-injective special biserial algebras, Comm. Algebra 22 (1994), no. 4, 1127-1160, DOI 10.1080/00927879408824898. MR.1261252 (95b:16008)

[22] Nicole Snashall and Rachel Taillefer, The Hochschild cohomology ring of a class of special biserial algebras, J. Algebra Appl. 9 (2010), no. 1, 73-122, DOI 10.1142/S0219498810003781. MR2642814(2011d:16009)

[23] Burkhard Wald and Josef Waschbüsch, Tame biserial algebras, J. Algebra 95 (1985), no. 2, 480-500, DOI 10.1016/0021-8693(85)90119-X. MR801283(87a:16056)

Institut DE MAThÉmatiques ET DE MOdÉlisation DE Montpellier I3M, UMR 5149,

Université Montpellier 2, F-34095 Montpellier cedex 5, France

E-mail address: Claude.Cibils@math.univ-montp2.fr 